\title{
Fetal Echocardiography:Short Term Profile from Shahid Gangalal National Heart Centre, Kathmandu, Nepal
}

\author{
Sudhir Regmi, ${ }^{1}$ Deewakar Sharma ${ }^{2}$ \\ ${ }^{1}$ Department of cardiology, National Academy of Medical sciences, Bir Hospital, Kathmandu,Nepal \\ ${ }^{2}$ Department of cardiology, Shahid Gangalal National Heart Centre, Bansbari, Kathmandu,Nepal \\ Corresponding Author: Sudhir Regmi. \\ Department of cardiology, National Academy of Medical sciences, Bir Hospital, Kathmandu, Nepal, E-mail:sudhirregmi@yahoo.com
}

\begin{abstract}
Background and Aims: Fetal echocardiography is helpful in early detection of Congenital Heart Disease.Our study was conducted to evaluate the most common indications of referral and outcome in a tertiary-care fetal echocardiography practice.

Methods: A Cross-sectional analysis of all pregnant women referred by obstetricians to cardiology unit for fetal echocardiography over a 1-year period (July 2014 and July 2015) was performed. The primary indications for referral for fetal echocardiography were obtained from the obstetric referral forms. Outcome data were extracted from performa containing client's demographic, physical examination and the fetal echocardiograhic data. Postnatal Echocardiography was advised to all cases having positive echocardiographic finding.

Results: A series of 251 fetal cardiac studies were reviewed. Average gestational age was 25.6 weeks (range, 18 to 38 weeks). Thirty-eight (15.1\%) pregnant women had abnormal fetal cardiac findings. The most common referral for fetal cardiac scan was related to maternal indications (48.6\%). Other indications were abnormal prenatal fetal findings in ultrasonography (23.1\%), family history of CHD (12\%), general screening (15.5\%), and follow up of IVF(In-vitro fertilization) (0.8\%). The highest yield of significant abnormal findings was there among patients referred with abnormal prenatal fetal finding in ultrasonography (47\%).

Conclusion: Majority of referral were for abnormal prenatal ultrasonographic findings. So, fetal echocardiography is an important part of overall management of the pregnancy at risk for producing an infant with congenital heart disease.
\end{abstract}

Key words: Congenital Heart Disease (CHD), Fetal Echocardiography, pregnancy.

\section{Introduction}

Congenital heart disease (CHD) is one of the most common congenital anomalies, and approximately half of infant deaths are due to CHD. ${ }^{1}$ The reported prevalence of moderate and severe CHDs in the United States is about 6 per 1000 live births, and the prevalence of CHDs, including mild forms, is 75 per 1000 live births. Studies report that the overall prevalence of CHDs is 8.1-18.7 per 1000 live births, and the prevalence of major CHDs (defects requiring intervention during the first year of life) is 3.0-4.4 per 1000 live births. The prevalence of congenital heart defects among fetuses is estimated to be even higher, at 14.6 per 1000 fetuses. Because complex congenital heart defects are common among fetuses and may result in spontaneous abortion or stillbirth, the total prevalence of congenital heart defects among stillbirths and live births may be higher than that reported among live births alone. ${ }^{2}$

Because early detection of CHD makes early medication, transcatheter intervention, or even surgery possible, prenatal diagnosis is considered essential. Since its introduction in 1964, prenatal diagnosis with fetal echo has improved the preoperative condition, morbidity, and mortality of patients with CHD. ${ }^{3}$ As the detection rates for congenital anomalies continue to increase, the demand for fetal echocardiography has grown. Accurate diagnosis of congenital heart disease via fetal echocardiography provides many benefits. It allows for a smooth transition between the pre- and post-natal states, with the opportunity to provide immediate care at birth, thereby avoiding the onset of hemodynamic compromise. ${ }^{4}$ Accurate diagnosis via fetal echocardiography allows for appropriate counseling to take place and for parents to take the opportunity to learn about the cardiac anomaly. This knowledge can allay parental fears, improve psychological state, and bolster coping skills in dealing with the birth of a child with life-threatening cardiovascular illness. ${ }^{5}$ The primary indications for fetal echocardiography, arise from routine prenatal sonography, a family history of congenital heart disease, maternal diabetes or systemic lupus erythematosus(SLE), fetal exposure to a teratogen, fetal karyotype abnormality, and other fetal system abnormalities. Despite the recognition of these risk factors, only $15-30 \%$ of cardiac defects are detected before birth. $^{7}$ 
Now the service of fetal echocardiography is provided only by Sahid Gangalal National Heart Center in Kathmandu, the capital city of Nepal for last 6 years. Since no study on fetal echocardiography from Nepal has been published yet, we aim to report our initial experience in this field.

\section{Methods:}

All cases referred for fetal echocardiography from July 2014 to July 2015 were included. Participants who denied informed consent were excluded from the study. A structured questionnaire was used for interview. The socio-demographic characteristics, height and weight of pregnant women were reported. Family history of CHD, previous obstretic history was inquired. Exposure to nonpregnancy related drugs and indication for referral were also noted. The examination was performed by using a PHILIPS ie33 /GE vividS6 /GE vivid7 echocardiography system with a convex and phase array probe having fundamental frequencies between 4 and $12 \mathrm{MHz}$.AIUM (American Institute of Ultrasound in Medicine) guideline ${ }^{8}$ was followed for completion of imaging data. The major scanning planes included long axis or four chamber view, trachea view, left and right outflow tracts and the major vessels view. Statistical analysis was done using SPSS version 20.

\section{Results:}

During the study period, we performed 251 echocadiographic examinations in the second or third trimester of pregnancy. The mean maternal age and the mean gestational age at the time of the initial prenatal echocardiographic examination were 28.8 years and 25.65 weeks, respectively. Majority of participants $(77.3 \%)$ were in age group of $20-35 y$ with $18.7 \%$ in above 35 yrs age group. Majority (75.7\%) of referral were done after 22 wks of gestation. Maternal condition accounted for the most common primary indication for referral (48.6\%). Abnormal cardiac findings $(23.1 \%)$ on prenatal sonography were the second most common indication of referral. Fetal indication included abnormal cardiac findings in USG screening, single umbilical artery, IUGR (Intra Uterine Growth Retardation) and fetal brady or tachycardia.

Other common indications for referral were a positive family history of congenital heart disease in $30(12 \%)$, screening for general purpose $39(15.5 \%)$ and follow up of fetus with history of IVF $(0.8 \%)$.Maternal Conditions leading to indication of fetal echo included maternal age ( $>35 \mathrm{yrs}$ ), gestational diabetes, hypertension, bad obstetric history, maternal viral illness in first trimester, exposure to drugs, history of arrhythmias and SLE in mother.

\section{Indications of referral for fetal echocardiography}

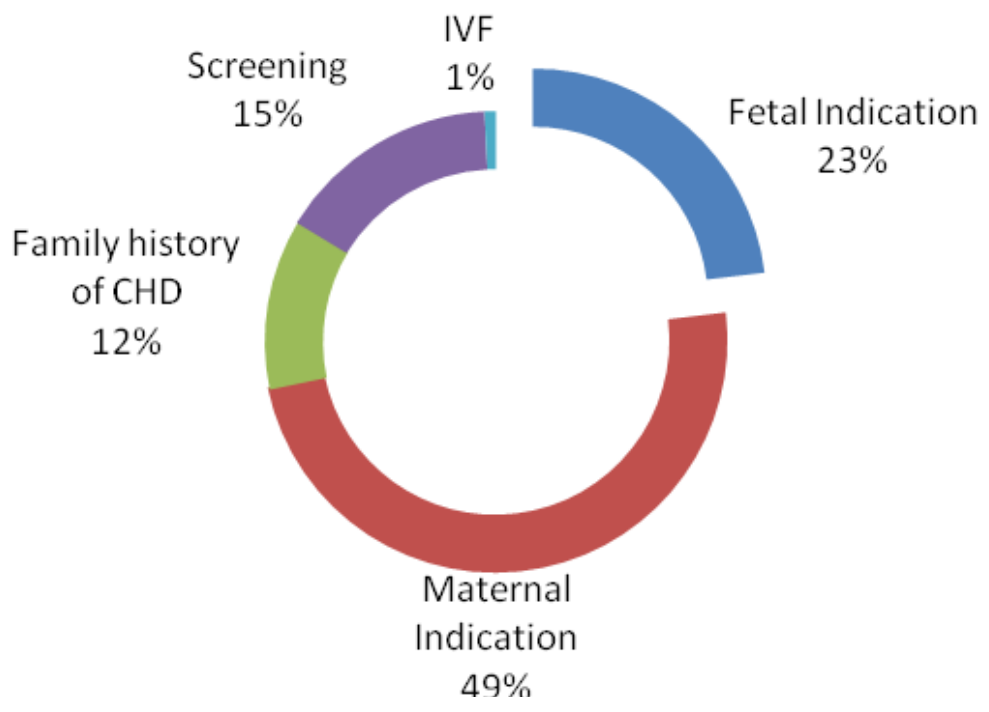

Fig 1: Indications of referral for fetal echocardiography.

Total of 38 fetal echocardiogram (15.1\%) were reported abnormal. The most common abnormal echocardiographic finding was small echogenic focus in left or right ventricle(7.6\%) followed by VSD(Ventricular Septal defect)(1.2\%), transient bradycardia (1.2\%), hypoplstic left ventricle $(0.8 \%)$, TAPVC(Total anomalous pulmonary vein connection $(0.8 \%)$,dysplastic tricuspid/mitral valve $(0.8 \%)$,single ventricle $(0.8 \%), \mathrm{AVCD}$ (Atrioventricular canal defect)(0.4\%),tricuspid atresia (0.4\%),DORV(Double outlet Right ventricle)( $0.4 \%)$,Pulmonary atresia( $0.4 \%)$ and eccentric hypertrophy of Left/Right ventricle( $0.4 \%)$. Out of 53 cases having fetal indication for echocardiography, 25(47\%) had some form of abnormal echo study. Whereas only 3 out of $122(2.4 \%)$ of cases with maternal indication showed positive findings on echocardiography. Most of the abnormal fetal echocardiographic findings were recorded in those mothers having first $(52.6 \%)$ and second $(44.7 \%)$ gravida.Out of 30 refferals having family history of congenital heart disease,2(6.6\%)echo study were positive for abnormal findings. 


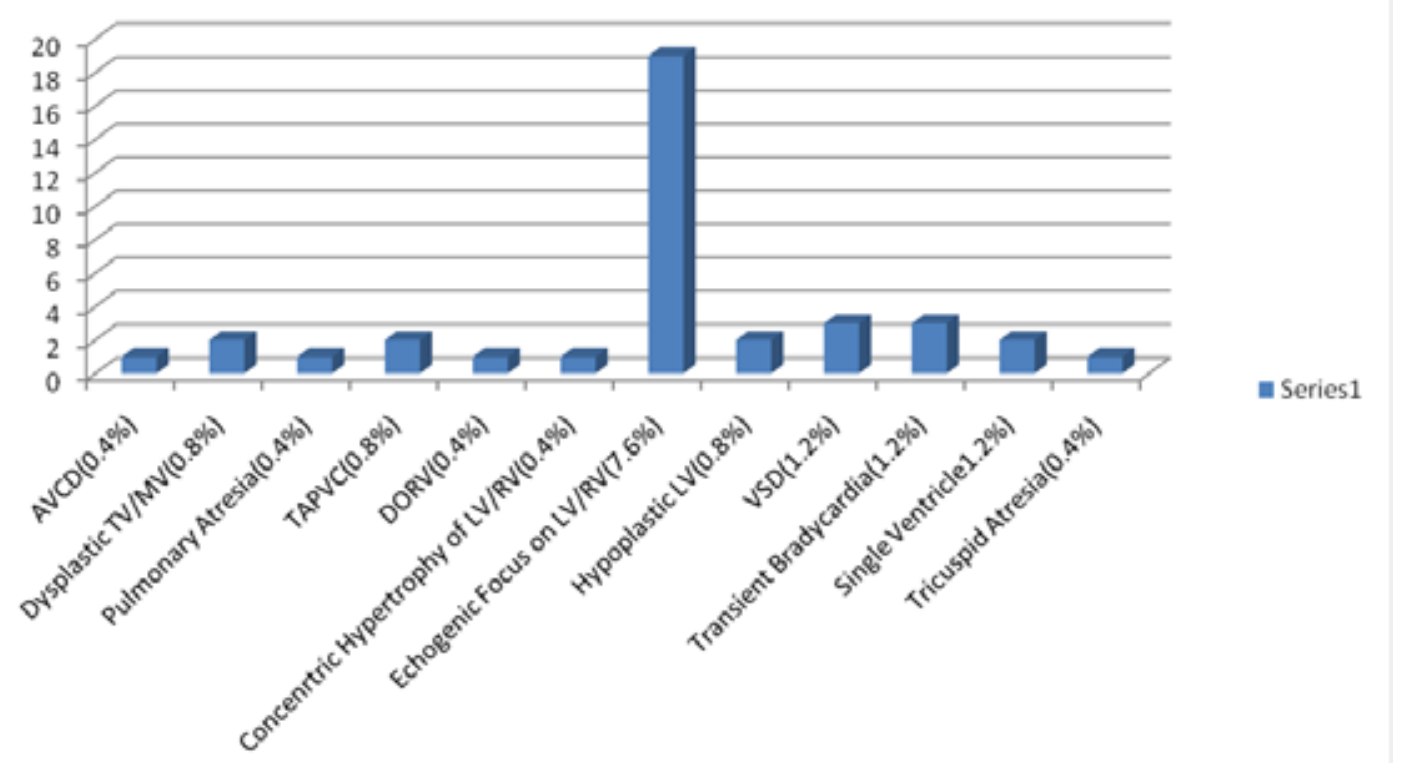

Fig 1: Findings in fetal echocardiography

\section{Discussion:}

Routine Obstetric ultrasound study doesn't detect cardiac abnormalities frequently. Moreover, ultrasonographers may not be familiar enough to focus upon specific cardiac finding on routine screening. Traditional risk factor for congenital heart disease such as maternal diabetes, family history of congenital heart disease, exposure to teratogens or radiation identify relatively few cases of congenital heart disease prenatally.,

In present study, majority (48.6\%) of total echocardiographic examination had been performed because of maternal condition which includes traditional risk factors with detection rate of only $2.4 \%$.Although screening patient with traditional risk factor is indicated, reports have been there of detecting most cardiac defect in low risk pregnancy. ${ }^{10}$ Suspected cardiac or noncardiac malformations on sceering sonography was the second most common indication for referral in our study. Similar referral patterns were reported by various authors. ${ }^{11,12,13}$ Our study confirmed or detected unsuspected cardiac defect in this group at the rate of $50 \%$.In a retrospective analysis by Cooper and colleague, $94 \%$ of fetal echocardiographic examination had been performed because a cardiac defect had been suspected on the basis of prenatal sonography, whose rate of detection was $68 \%$.In other studies, $4-5 \%$ of echo exam was done on the basis of abnormal screening prenatal sonography with CHD confirmed in $38-50 \%$ cases $^{14,15,16}$

Out of all abnormal echo findings, small echogenic focus in ventricle $(50 \%)$ surpasses all other finding in our study. We could not prove significance of this finding but interestingly all infant screening echocardiography at 6 months of age showed resolution of the foci. Study done by Josheph $\mathrm{R}$ et $\mathrm{al}^{17}$ has demonstrated that biventricular intracardiac echogenic foci are more frequently associated with aneuploidy.Moreover, study done by Ali MK et al has included this finding as soft markers of aneuploidy in second trimester fetuses. ${ }^{18}$

Although proper timing of referral for fetal echocardiography is between 18-22 weeks of gestation, majority $(75.7 \%)$ of referral we received were after $22 \mathrm{wks.} .^{19}$ the reason might be due to unavailability of fetal echocardiography in multi speciality hospitals from where our study population were referred. Moreover, lack of awareness of proper timing of fetal echocardiography also plays an important role in delayed study. Major limitation of our study is lack of proper follow up of positive echo finding cases in neonatal period. We don't have autopsy report after termination of pregnancy too. Although majority of fetuses showing echogenic intracavitary foci showed resolution in follow up study, our opinion to this entity is limited by non availability of genetic testing in our setup. Another aspect of our study is non representative sample to conclude upon prevalence in general population because all of them were referred from multispecialty hospitals and clinics.

Universal screening for congenital heart disease at the time of routine prenatal sonographic examination might be the most effective approach to improving the rate of prenatal detection of serious cardiac anomalies. The American College of Obstetricians and Gynecologists ${ }^{20}$ and the American Institute of Ultrasound in Medicine ${ }^{21}$ recommend that the 4-chamber view be evaluated during routine prenatal sonographic examinations performed in the second and third trimesters. It is estimated that with appropriate training and experience, sonographers evaluating the 4-chamber view of the fetal heart can identify 40 $50 \%$ of cases of congenital heart disease and that adding views of the ventricular outflow tracts can increase the detection rate to $60-80 \% . .^{22}$

\section{Conclusion}

With various limitations, current study shows different patterns of referral for fetal echocardiography in our setup. Moreover, various findings suggest towards use of this technology in normal as well as abnormal cardiac results on prenatal sonography. So, Strong referral system with facility for postnatal follow up echocardiography and postmortem study of aborted fetuses is also required for proper outcome study on fetal echocardiography. 


\section{References:}

1. Hoffman JI, Kaplan S. The incidence of congenital heart disease. J Am Coll Cardiol 2002; 39:1890-900.

2. Zhang Y,Colarusso R T, Correa A.Observed Prevalence of Congenital Heart Defects From a Surveillance Study in China. J Ultrasound Med 2011; 30:989-995

3. Verheijen PM, Lisowski LA, Stoutenbeek P, Hitchcock JF, Brenner JI, Cope JA, et al. Prenatal diagnosis of congenital heart disease affects preoperative acidosis in the newborn patient. J Thorac Cardiovasc Surg 2001; 121:798.

4. Rychik J, Ayres N, Cuneo B. American Society of Echocardiography Guidelines and Standards for Performance of the Fetal Echocardiogram.J Am Soc Echocardiogr 2004;17:803-10.

5. Sklansky M, Tang A, Levy D, Grossfeld P, Kashani I, Shaughnessy R, et al. Maternal psychological impact of fetal echocardiography. J Am Soc Echocardiogr 2002; 15:159-66.

6. Cheitlin MD, Armstrong WF, Aurigemma GP, et al. ACC/AHA/ASE 2003 guideline update for the clinical application of echocardiography-summary article: a report of the American College of Cardiology/American Heart Association Task Force on Practice Guidelines(ACC/ AHA/ASE Committee to Update the 1997 Guidelines for the Clinical Application of Echocardiography). J Am Coll Cardiol 2003; 42:954

7. Grandjean H, Larroque D, Levi S. The performance of routine ultrasonographic screening of pregnancies in the Eurofetus Study. Am J Obstet Gynecol 1999; 181:446.

8. American Institute of Ultrasound in Medicine. AIUM practice guideline for the performance of fetal echocardiography. J Ultrasound Med 2013; 32: 1067-1082.

9. Cooper MJ, Enderlein MA, Dyson DC, et al. Fetal echocardiography: retrospective review of clinical experience and an evaluation of indications. Obstet Gynecol $1995 ; 86: 577$.

10. Roodpeyma S, Kamali Z, Afshar F, et al. Risk factors in congenital heart disease. Clin Pediatr (Phila) 2002; 41:653.

11. Achiron R, Glaser J, Gelernter I, et al. Extended fetal echocardiographic examination for detecting cardiac malformations in low-risk pregnancies. BMJ 1992; 304:671.

12. Allan LD, Sharland GK,Milburn A, et al. Prospective diagnosis of 1,006 consecutive cases of congenital heart disease in the fetus. J Am Coll Cardiol 1994; 23:1452.

13. Davis GK, Farquhar CM, Allan LD, et al. Structural cardiac abnormalities in the fetus: reliability of prenatal diagnosis and outcome. Br J Obstet Gynaecol 1990;97:27

14. Bader RS.Abnormal cardiac findins in prenatal sonographic examination:an important indication for fetal echocardiography? Journal of the Saudi Heart Association 2008;20:29-33.

15. Wheller JJ, Reiss R, Allen HD. Clinical experience with fetal echocardiography. Am J Dis Child 1990; 144:49.

16. Copel JA, Pilu G, Green J, Hobbins JC, Kleinman CS. Fetal echocardiographic screening for congenital heart disease: the importance of the four-chamber view. Am J Obstet Gynecol 1987; 157:648-655.

17. Smythe JF, Copel JA, Kleinman CS. Outcome of prenatally detected cardiac malformations. Am J Cardiol 1992; 69:1471.

18. Joseph R. Wax, Christine P. Fetal Intracardiac Echogenic Foci: Does It Matter Which Ventricle? The American Institute of Ultrasound in Medicine.J Ultrasound Med 17:141-144, 1998.

19. Ali KM, Shazly SA, Ali AH. Ultrasonographic soft markers of aneuploidy in second trimester fetuses. Middle East Fertility Society Journal 2012; 17:145-151.

20. Rychik J, Ayres N, Cuneo B.American Society of Echocardiography Guidelines and Standards for Performance of the Fetal Echocardiogram. J Am Soc Echocardiogr 2004;17:803-10.

21. American College of Obstetricians and Gynecologists. Ultrasonography in pregnancy (Technical Bulletin No. 187). Washington, DC: ACOG; 1993.

22. American Institute of Ultrasound in Medicine. Guidelines for the performance of the antepartum obstetrical ultrasound examination. J Ultrasound Med 1991; 10:576.

22. Small M, Copel JA. Indications for fetal echocardiography. Pediatr Cardiol. 2004;25(3):210-22.
Cite this article as: Sudhir Regmi,Deewakar Sharma. Fetal Echocardiography:Short Term Profile from Shahid Gangalal National Heart Centre, Kathmandu, Nepal. Nepalese Heart Journal 2016; 13(1):9-12. 